\title{
Case report - Primary renal echinococcal infection
}

Jennifer Bjazevic ${ }^{1}$; Dor Golomb ${ }^{1}$; Michael S. Silverman ${ }^{2}$; Stephen E. Pautler ${ }^{1}$; Hassan Razvi ${ }^{1}$ ${ }^{1}$ Division of Urology, Department of Surgery, University of Western Ontario, London, ON, Canada; ${ }^{2}$ Division of Infections Diseases, Department of Medicine, University of Western Ontario, London, ON, Canada

Cite as: Can Urol Assoc J 2020 March 23; Epub ahead of print. http://dx.doi.org/10.5489/cuaj.6394

Published online March 23, 2020

$* * *$

\section{Abstract}

Renal echinococcal infection is an uncommonly encountered infection in North America but is endemic in many parts of the world. With increasing migration, it is conceivable that practicing Canadian physicians will see more patients presenting at various stages of infection. Herein, we describe an unusual presentation of primary renal echinococcal infection and describe current diagnostic, as well as medical and surgical management strategies. 


\section{Introduction}

Echinococcus is a parasitic infection due to the cestode, Echinococcus granulosus, and is endemic in the Middle East, China, Eastern and Sub-Saharan Africa, South and Central America, New Zealand, Australia and Alaska. ${ }^{1}$ The dog is the definitive host, with the adult Echinococcus worm residing in the proximal jejunum mucosa. Sheep are the intermediate host, and humans become infected through oral ingestion of parasite eggs in contaminated water, soil, vegetables, or from direct contact with the animal hosts. ${ }^{2}$ Once ingested, the embryo exits the egg, penetrates the human intestinal mucosa and enters the blood circulation. ${ }^{3}$ Systemic dissemination through the portal venous and lymphatic systems most commonly affects the liver (75\%) and lungs $(15 \%)$; however, infection may affect almost any anatomical location. ${ }^{4}$ Primary renal echinococcal infection, is rare, accounting for only $2-3 \%$ of cases. ${ }^{5}$

Renal involvement usually occurs in the form of hydatid cysts. Most patients with primary renal involvement remain asymptomatic. ${ }^{4}$ Over time, the cysts enlarge and may be associated with abdominal pain due to hydatiduria, fever, hematuria, abdominal mass and hypertension. ${ }^{6}$ Hydatiduria is reported to occur in $10-20 \%$ of cases due to rupture of the cysts into the collecting system and results in the passage of whitish material in the urine. ${ }^{6}$ Rarely cyst rupture can occur, resulting in anaphylaxis. ${ }^{7}$ Herein, we describe a rare case of primary renal echinococcal infection presenting in Canada with hydatiduria.

\section{Case report}

A 34-year-old man was referred for evaluation of an ultrasound finding of a complex right renal cyst (Figure 1A). He had been granted refugee status and immigrated to Canada 4 months prior. He had emigrated from Lebanon but was born in Syria and had lived there until age 15. While living in Syria, he had worked as a shepherd. In his mid-twenties, he reported undergoing an open surgical procedure on his kidney but could not relay the reason, nor were there any medical records available. He did not recollect receiving any prolonged courses of antibiotics and denied a history of tuberculosis.

A 3-phase, contrast-enhanced CT-scan of the abdomen was performed revealing a right thick-walled, upper pole cortical renal cyst, without enhancement, consistent with a Bosniak $2 \mathrm{~F}$ lesion. There were no signs of any calculi, hydronephrosis or other organ findings (Figure 1B). At follow-up 6 months later, he remained asymptomatic and an ultrasound revealed a stable cyst.

Three months later, he presented to the emergency department complaining of new onset, intermittent right flank pain. A non-contrast CT revealed new right hydroureteronephrosis to the bladder, without any ureteric or renal calculi. He denied fever, hematuria or seeing a stone pass, but had noted some brownish colored debris in his urine.

Urine microscopy demonstrated pyuria, without any evidence of bacteria; and urine culture and cytology were unremarkable. Urine for acid fast bacilli and mycobacteria were 
negative, a tuberculosis (TB) skin test was non-reactive, and chest $\mathrm{x}$-ray was normal. Complete blood count $(\mathrm{CBC})$ revealed a mild eosinophilia $\left(0.9 \times 10^{9} / \mathrm{L}\right)$, and serum creatinine was normal.

Retrograde pyelography revealed a large, irregular shaped filling defect in the right upper pole (Figure 2A). Flexible nephroscopy revealed whitish amorphous-looking material adherent to the urothelium, and biopsies were taken and sent for pathology (Figure 2B).

The pathology of the amorphous material was compatible with fragments of urothelium, with underlying mixed chronic inflammation and abundant eosinophils. No granulomas, dysplasia or malignancy were present. The urothelial biopsy consisted of predominantly acellular eosinophilic material with a laminated appearance and thin ribbons of densely eosinophilic granular material. No organisms were identified. The pathological diagnosis was suspicious for echinococcal infection.

The patient was referred to Infectious Disease. Echinococcal species IgG enzyme- linked immunoassay (EIA) serology was found to be to be strongly reactive (IgG EIA Index: 10.19; cutoff for Reactive $>1.1$ ), indicating detectable levels of echinococcal antibodies. Abdominal ultrasound demonstrated no liver involvement.

Surgical therapy was recommended for definitive management of his Echinococcal disease. Given his prior open surgery, with what seemed to be incomplete excision of the hydatid cyst, total nephrectomy was recommended. He was prescribed oral albendazole (400mg twice daily) as a suppressive anthelmintic 1 week preoperatively. Open right nephrectomy through a subcostal incision was performed. Care was taken to successfully avoid rupturing the cyst during the dissection.

Pathological examination of the kidney specimen revealed a cystic lesion with a thick fibrous wall, with an abundant chronic inflammatory process, composed of lymphocytes, plasma cells, occasional multinucleated giant cells and eosinophils. Within the cyst there was a residual inner acellular layer, with a prominent laminated appearance and an overlying eosinophilic germinal layer. No protoscolices (parasitic larvae) were seen. The features were in keeping with a treated echinococcus cyst (Figure 3).

His post-operative course was unremarkable, and he was maintained on suppressive albendazole for an additional 1- month post-operatively. Follow-up abdominal ultrasound did not demonstrate any evidence of daughter cysts and his eosinophilia resolved. Both ultrasound and CT imaging have a high sensitivity and specificity $(>90 \%)$ for detection of daughter cysts. ${ }^{8}$

\section{Discussion}

The diagnosis of echinococcal infection may be difficult to establish even in areas where the condition is endemic. The clinical course may be variable, and imaging techniques may assist in the diagnosis, but are occasionally inconclusive. Eosinophilia suggests parasitic infection but is not specific. Serological testing is helpful especially for liver involvement, although may be much less sensitive where other organs are involved. Sensitivity and specificity of newer 
commercial Echinococcal IgG assays are reported to be as high as $95.2 \%$ and $94.3 \%$ respectively. ${ }^{9}$ False positive serology is an issue due to cross reactivity with antigens from other helminths. ${ }^{10}$ Therefore diagnosis remains heavily reliant on clinical suspicion.

Various treatments have been described for the treatment of renal echinococcal infection including percutaneous intervention, open surgical excision, laparoscopic surgery and medical treatment. ${ }^{11}$ However, definitive treatment remains surgical and requires removal of the entire intact cyst, in order to reduce the risk of disease recurrence and prevent anaphylaxis with cyst rupture. ${ }^{12}$ While traditionally an open retroperitoneal approach has been recommended to avoid contamination of the peritoneal cavity, minimally invasive techniques have been described. ${ }^{12}$ When possible a nephron-sparing approach is preferred; however, in cases where the kidney is poorly functioning, there is hydatiduria, or when partial nephrectomy is not feasible, removal of the entire kidney is the treatment of choice. ${ }^{12}$ Puncture aspiration-injection-re-aspiration (PAIR) performed under imaging guidance in association with systemic medical therapy has been quite effective in patients with hepatic hydatid disease, but experience in renal hydatid disease has been quite limited and is generally restricted to non-operable patients. ${ }^{13}$

Medical management with anthelminthics alone has been reported to achieve poor results, due to the drugs' low penetration into the cyst. These agents are also not without potentially serious adverse effects, such as hepatotoxicity, leukopenia and allergic reactions. ${ }^{14}$ The main role for medical management is in conjunction with surgery, where the anthelminthics have been used in order to reduce the size of cysts, sterilise them, and to prevent relapses. ${ }^{15}$ Addition of praziquantel to albendazole has been recommended by some authors but again the evidence for this approach remains quite limited. ${ }^{15}$

\section{Conclusions}

Although a rare condition in North America, echinococcal infections are common in many other parts of the world. With population migration, it is conceivable that more patients will be seen outside endemic areas. While primary renal involvement is rare, Canadian urologists should be aware of appropriate diagnostic testing and recommended therapies. 


\section{References}

1. Vuitton DA. The WHO informal working group on echinococcosis. The coordinating board of the WHO-IWGE. Acta Trop 1997;67:147-8.

2. Fazeli F, Narouie B, Firoozabadi MD, et al. Isolated hydatid cyst of kidney. Urology 2009;73:999-1001. Available at: http://www.ncbi.nlm.nih.gov/pubmed/19193416, accessed November 6, 2019.

3. Sayilir K, Iskender G, Oğan C, et al. A case of isolated renal hydatid disease. Int J Infect Dis 2009;13:110-2.

4. Bandyopadhyay A, Khatua S, Das S, et al. A rare case of primary renal hydatid cyst presenting with hydatiduria. J Parasit Dis 2015;39:577-80. Available at: http://www.ncbi.nlm.nih.gov/pubmed/26345075. Accessed November 6, 2019.

5. Kumar SA, Shetty A, Vijaya C, et al. Isolated primary renal echinococcosis: A rare entity. Int Urol Nephrol 2013;45:613-6. Available at: http://www.ncbi.nlm.nih.gov/pubmed/23435774. Accessed November 6, 2019.

6. Kundu A, Mongha R, Narayan S: Primary hydatid cyst of kidney and ureter with gross hydatiduria: A case report and evaluation of radiological features. Indian J Urol 2008; 24:116.

7. Neumayr A, Troia G, de Bernardis C, et al: Justified concern or exagerrated fear: The risk of anaphylaxix in percutaneous treatment of cystic echinococcosis: A sytematic literature review. PLoS Negl Trop Dis 2011;5:e1154.

8. Mehta P, Prakash M, Khandelwal N. Radiological manifestations of hydatid disease and its complications. Trop Parasitol 2016;6:103-12.

9. Public Health Ontario. Parasitic serology update - Test methodology change 2017. https://www.publichealthontario.ca/-/media/documents/lab/lab-sd-117-parasitic-serologytest-method-change.pdf?la=en. Accessed Nov 27, 2019.

10. Fathi S, Jalousian F, Hosseini S, et al. A study of cross-reactivity between recombinant EPC1 antigen of Echinococcus granulosus in serum from patients with confirmed cystic echinococcosis infection and other parasitic infections. Am J Trop Med Hyg 2016;94: 1313-17.

11. Aggarwal S, Bansal A: Laparoscopic management of renal hydatid cyst. JSLS J Soc Laparoendosc Surg 2019;18:361-6. Available at: http://www.ncbi.nlm.nih.gov/pubmed/24960508. Accessed November 6, 2019.

12. Paramythiotis D, Bangeas P, Kofina K, et al. Presence of an isolated hydatid cyst in the left kidney: Report of a case of this rare condition managed surgically. Case Rep Urol 2016;doi:10.1155/2016/6902082.

13. Rexiati M, Mutalifu A, Azhati B, et al. Diagnosis and surgical treatment of renal hydatid disease: a retrospective analysis of 30 cases. PLoS One. 2014;9:e96602.

14. Von Sinner WN, Hellström M, Kagevi I, et al. Hydatid disease of the urinary tract. J Urol 1993;149:577-80. Available at: http://www.ncbi.nlm.nih.gov/pubmed/8437269. Accessed November 11, 2019.

15. Velasco-Tirado V, Alonso-Sardón M, Lopez-Bernus A, et al. Medical treatment of cystic echinococcosis: Systematic review and meta-analysis. BMC Infect Dis 2018;18:306. 


\section{Figures and Tables}

Fig. 1. (A) Ultrasound images of complex right renal cyst: (i) transverse view; (ii) sagittal view; (iii) sagittal view with doppler flow. (B) Triphasic computed tomography images of complex right renal cyst: (i) selected non-contrast axial image; (ii) selected arterial and urogram phase axial image; (iii) selected non-contrast coronal image; (iv) selected arterial and urogram phase coronal image.

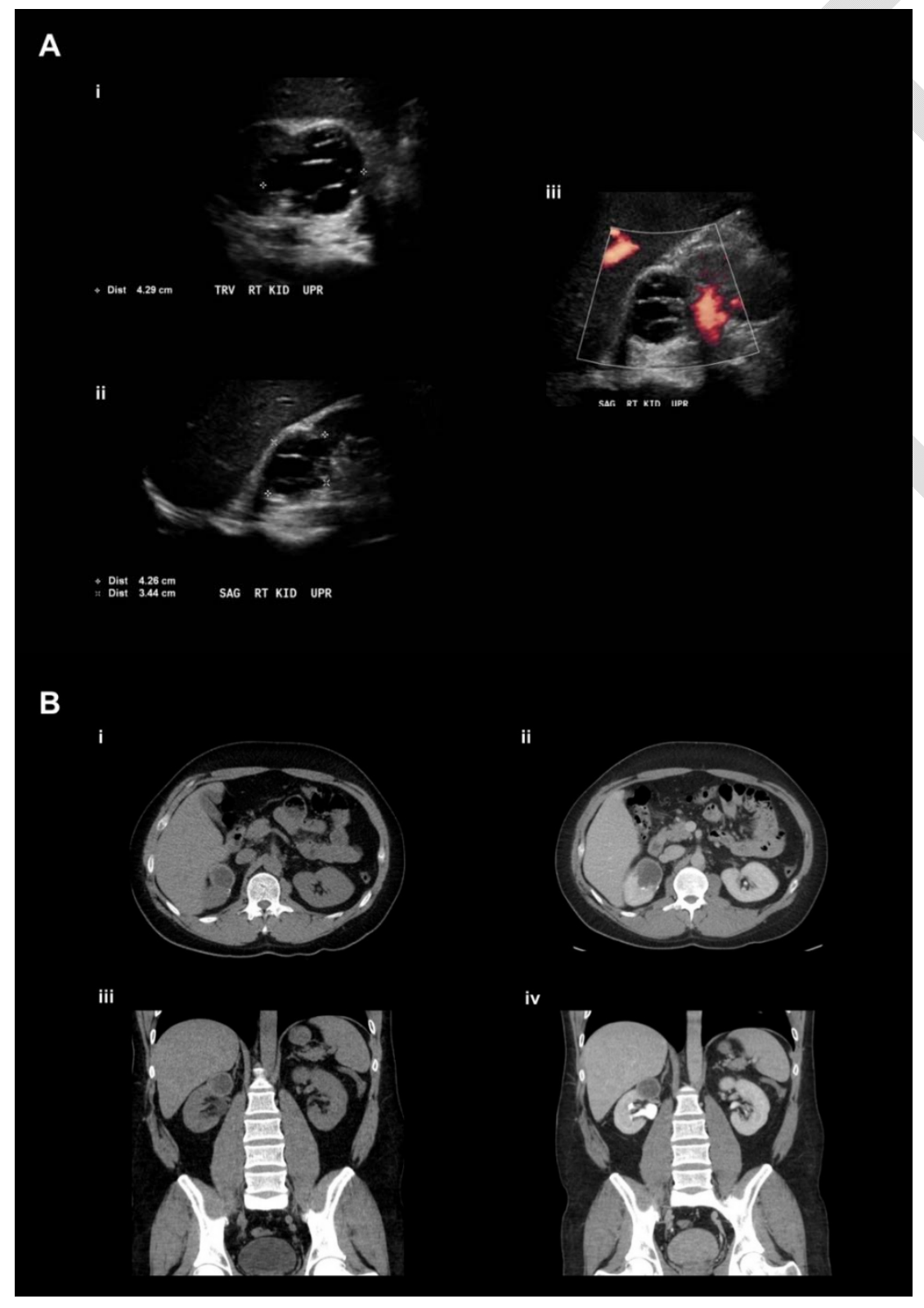


Fig. 2. (A) Retrograde pyelogram demonstrating large filling defect in the upper pole calyces. (B) Selected images from flexible ureteroscopy demonstrating amorphous material adherent to the mucosa within the upper pole and an erythematous appearance of the surrounding mucosa.

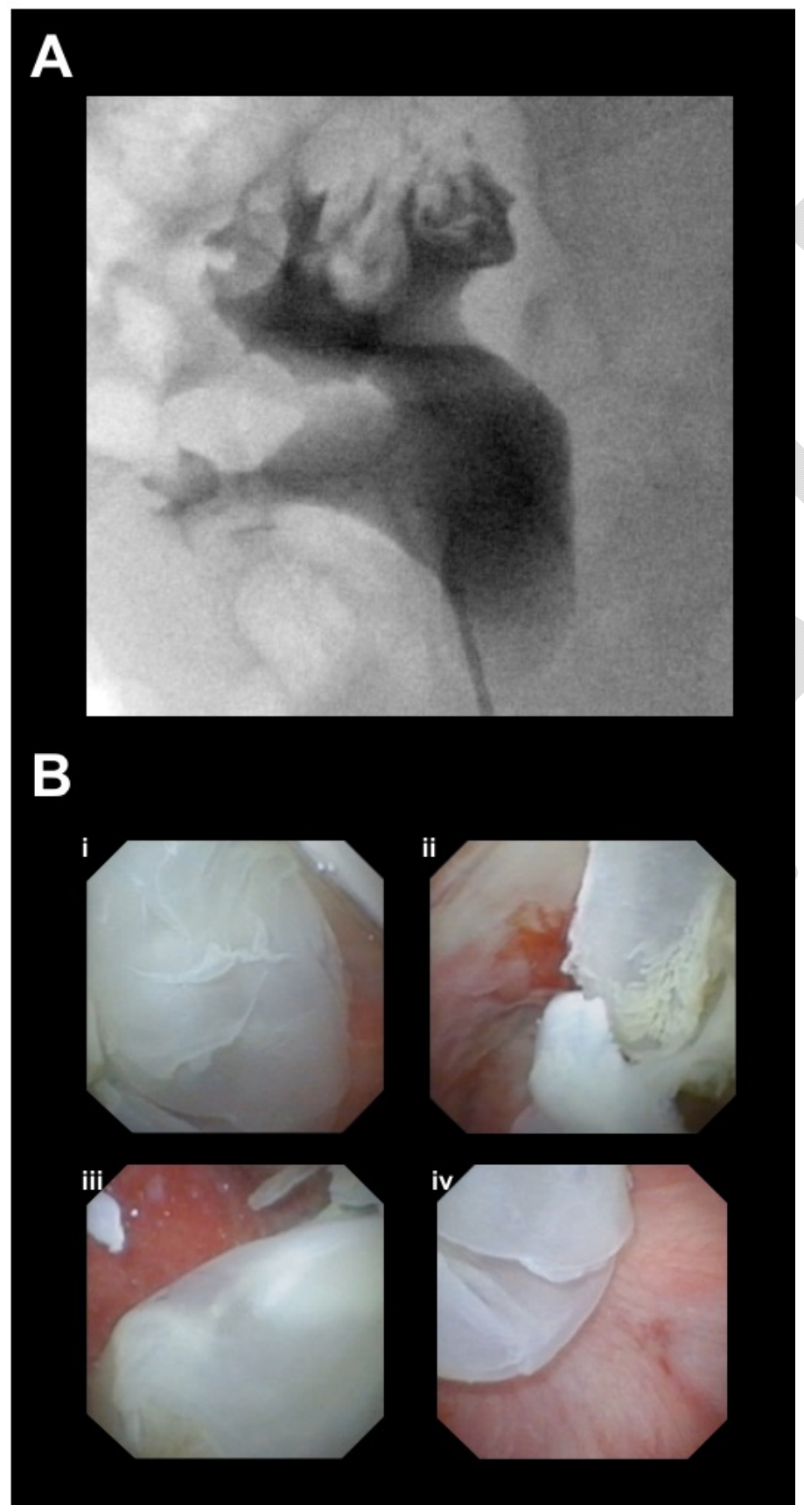


Fig. 3. (A) Pathology images from radical nephrectomy specimen; and (B) gross specimen bivalved with $1 \mathrm{~cm}$ scale bar. (B) Gross specimen of echinococcal cyst bivalved with $1 \mathrm{~cm}$ scale bar. (C) Microscopy images with hematoxylin and eosin stain under 4X magnification with 100 micron scale bar. (D) Microscopy images with hematoxylin and eosin stain under 4X magnification with 100 micron scale bar.

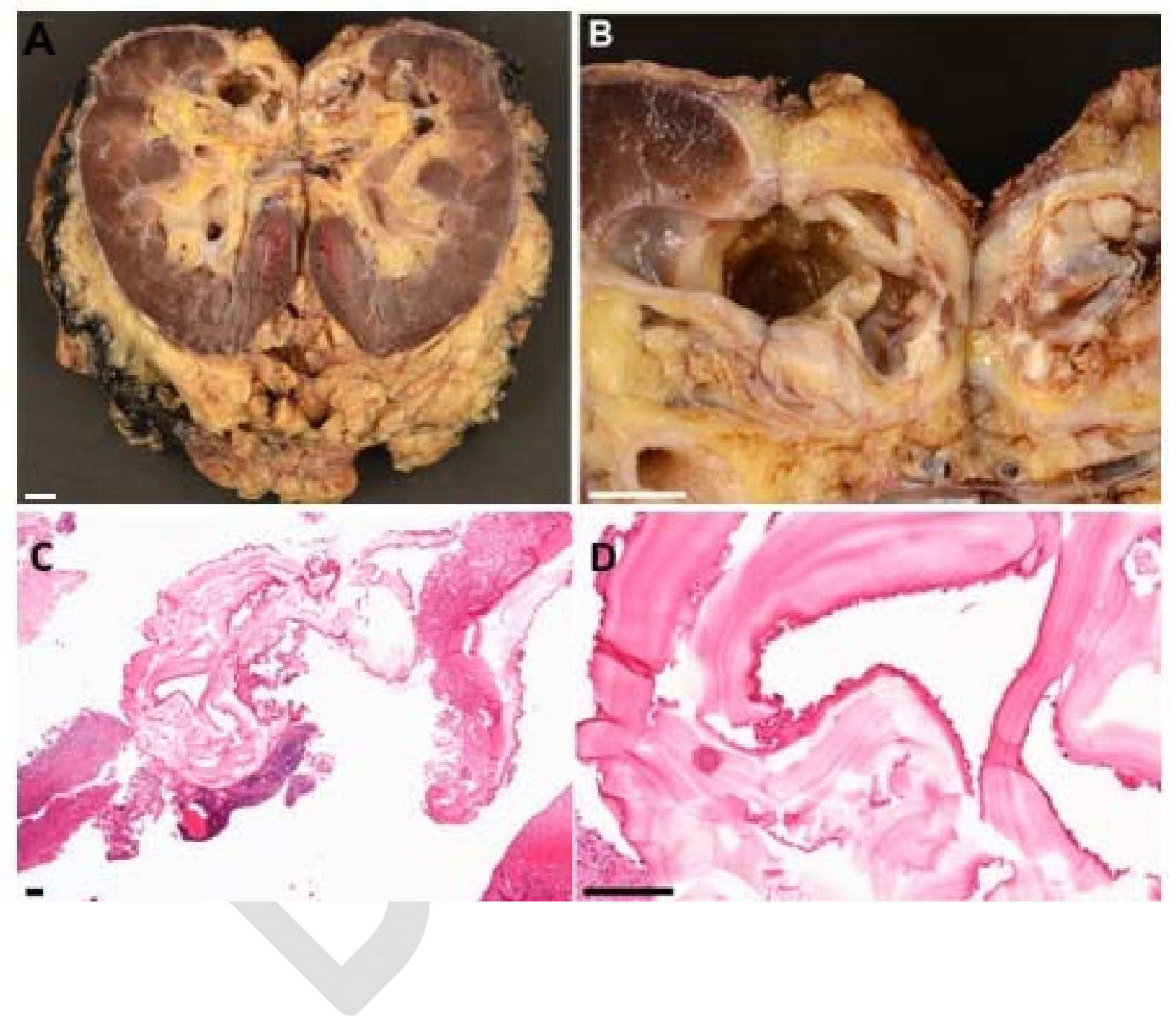

